\title{
LA EDUCACIÓN DE LAS MUJERES EN IBEROAMÉRICA. ANÁLISIS HISTÓRICO
}

Por Teresa González Pérez (coord.). Valencia, Tirant humanidades: márgenes, 2020, 812 páginas. ISBN: 978-84-17973-20-9.

Desde diferentes puntos del planeta, en las últimas décadas, las mujeres han tratado de reconstruir su historia. El sistema androcéntrico que todavía impera en nuestras sociedades las ha mantenido históricamente en la periferia, la exclusión y el olvido, por lo que su historia ha sido borrada u olvidada de manera sistemática. En el libro La educación de las mujeres en Iberoamérica. Análisis histórico, autoras de diferentes nacionalidades, contextos y trayectorias tratan de recuperar la historia de la educación de las mujeres, así como las aportaciones y la influencia ejercida por ellas a través de los siglos en España y Latinoamérica. Las investigaciones a este respecto son cada vez más numerosas y este libro ofrece un compendio de diferentes temáticas y contribuciones para la reivindicación de la historia desde una perspectiva de género. Así, se adentra en la educación femenina en sus diferentes niveles a través de los siglos y sus referentes olvidados. El libro se estructura en tres partes diferenciadas: una primera parte que se corresponde con los siglos XVIII y XIX, una segunda que contiene los textos relativos a la primera mitad del siglo XIX, y una tercera que recoge desde la segunda mitad del siglo XX a los albores del siglo XXI.

A modo de introducción, Teresa González Pérez realiza un primer acercamiento a la naturaleza y el sentido de la educación de las mujeres. La coordinadora del libro destaca y celebra el aumento de estudios relacionados con la historia de la educación de las mujeres de manera progresiva desde los años ochenta del pasado siglo. Tal y como ella explica, las propias mujeres han rescatado la memoria educativa de sus iguales, han dado voz a las «sin voz», se han proclamado protagonistas de la historia y se han enfrentado a las normas patriarcales imperantes. Este 
libro pone de manifiesto, una vez más, esta realidad. A través de diecinueve textos, relacionados con la educación femenina, se exponen diferentes realidades históricas en diversos espacios, contextos y niveles.

El primer capítulo escrito por Ana Armenta-Lamant Deu, «La educación femenina de las clases populares durante la Ilustración española: ¿enseñanza o adoctrinamiento?», versa sobre la educación de las mujeres de clase obrera a lo largo del siglo XVIII. La autora nos acerca tanto a la realidad de las maestras como a la de las alumnas y explica cómo la educación femenina no preocupaba a gobernantes y eclesiásticos. Así, los avances que se dieron fueron mucho menores a los del resto de Europa por el arraigo a los valores tradicionales, el ideal de mujer defendido, la estrecha relación entre la educación y la Iglesia católica y la falta de medios destinados a la formación de las mujeres. Sin embargo, a pesar del alto absentismo y analfabetismo, existieron casos en los cuales la educación resultó fundamental para la mejora social de las mujeres.

En el segundo texto, «Modelo educativo para las mujeres españolas en el siglo XIX. Reforzando el arquetipo tradicional», Teresa González Pérez analiza la formación del carácter de las mujeres a través de la educación en las Islas Canarias desde el año 1857 con la promulgación de la Ley Moyano. La autora profundiza en el caso de esta región ultraperiférica de España por sus características específicas en ese momento. La situación económica, política y social era más precaria, la tasa de analfabetismo mayor y el acceso de las niñas a la educación menor, mientras que los representantes públicos eran negligentes en este sentido. A pesar de ello, a lo largo del artículo se recoge cómo proliferaron publicaciones de mujeres que hablaban de la educación de las mujeres y de su ideal de mujer. Las mujeres se reivindicaron y comenzaron a ocupar el espacio público a través de su participación en congresos a favor de la educación de las mujeres.

En tercer lugar, Adelina Arredondo habla sobre la nueva concepción de la mujer, la modernidad, la alfabetización de las niñas y la feminización del magisterio en el momento de construcción del Estado Independiente Mexicano en el capítulo «Cómo fue emergiendo y cómo fue sedimentándose y normalizando el oficio femenino de enseñar. El caso de México». En este contexto, Adelina habla sobre las «amigas» —maestras que cuidaban a niñas de entre 3 y 10 años—, la importancia 
de las congregaciones religiosas para la educación de las mujeres, las desigualdades existentes, el ideal de mujer del momento y el papel de la educación rural para la feminización del magisterio dadas las condiciones precarias, el bajo sueldo y las exigencias de esta enseñanza. La educación rural mejoró la alfabetización significativamente y contribuyó a la generalización de la profesión de maestra en el marco de la creación del Estado Independiente.

El capítulo titulado «Las escuelas de señoritas de Gran Canaria: síntesis de una formación de adorno» trata la enseñanza de las lenguas extranjeras en la formación de las mujeres de clase acomodada. Las Islas Canarias han contado históricamente con unas características propias por su desarrollo de la economía en relación con turistas y comerciantes internacionales. Así, a través del Reglamento del Colegio de Señoritas de Nuestra Señora de Rosario, María Jesús Vera-Cazorla analiza cuestiones tales como el modelo de mujer que definían estas escuelas en el siglo XIX y el currículum que seguían o su organización, deteniéndose en la enseñanza de idiomas como un elemento de distinción de clase.

En «Del hogar al colegio y del colegio al hogar y a la calle. Acceso a la educación e identidades de género: el Colegio Superior de Señoritas (Costa Rica, 1888-1940)», Eugenia Rodríguez Sáenz incide en la construcción de la identidad de las mujeres como madres, esposas y formadoras de ciudadanos. En este colegio, al cual también accedían alumnas pobres a través de un sistema de becas, se fundó la Liga Feminista en 1923. Un año antes, con las Campañas Sanitarias de 1922, las mujeres adquirieron el rol de educadoras sociales. Se les formó en hábitos de urbanidad, moral e higiene como promotoras de las políticas higienizadoras del Estado, lo que permitió a las mujeres contar con visibilidad pública y adquirir un papel como agentes activos de la transformación social.

El texto que cierra la primera parte del libro es «La educación y participación de las mujeres en las revistas pedagógicas» de Teresa González Pérez y Soledad Montes Moreno. En este capítulo se emplea una doble metodología cualitativa y cuantitativa para analizar la presencia femenina tanto en la participación como en el eje temático de la revista La Escuela Moderna. A lo largo del siglo XIX muchas personas ilustres entendieron que la educación de las mujeres era fundamental para el 
progreso social y económico del país, lo cual quedó patente con el surgimiento en 1891 de la revista La Escuela Moderna. En esta revista escribieron maestras y profesoras sobre la educación de las mujeres, se recuperaron referentes femeninos, se acercaron a la situación de la educación femenina en otros países y, en definitiva, convirtieron la educación de las mujeres en objeto de estudio y discusión.

La segunda parte, centrada en la primera mitad del siglo XX, comienza con el capítulo titulado «Carmen Mondragón versus Nahui Olin, metarelato de la educación mexicana en los años veinte y treinta del siglo XX». En él, Guadalupe Sánchez Álvarez realiza un repaso por la vida de Carmen Mondragón desde su niñez en París, donde escribió A los diez años sobre mi pupitre, hasta la figura de Nahui Olin como mujer transgresora, rompedora de normas y precursora de la liberación sexual femenina, pasando por su mudanza a México o su boda y separación. A través de este texto, la autora nos presenta a una mujer que fue juzgada y cuya historia trató de ser borrada tras su muerte por no ceñirse a los roles y espacios que le correspondían por su situación socio-económica.

El sexto capítulo trata sobre la figura y obra de Júlia Lopes de Almeida. A través de cuatro de sus textos (A falência, A intrusa, Cruel amor y Correiro da roça), Eurídice Hespanhol Macedo Pessoa analiza la educación de las mujeres brasileñas a inicios del siglo XX. En este capítulo, «La educación para las mujeres brasileñas a inicios del siglo XX en las páginas de las novelas de Júlia Lopes de Almeida», Pessoa nos muestra la visión de esta autora que militó activamente en las sociedades femeninas, participó en la creación de la Academia Brasileira de Letras-ABL, y, a pesar de los impedimentos que tuvo por el hecho de ser mujer, escribió más de cuarenta obras. A través de cuentos, textos para teatro, literatura infanto-juvenil y crónicas, habló de mujeres con realidades muy diferentes y planteó la necesidad de transformar su educación.

A continuación, «La educación arqueo-antropológica argentina en perspectiva de género: comienzos del siglo $\mathrm{XX}$ » indaga sobre el desarrollo de esta educación a través de Antonia Juliane Dillenius. El artículo se centra en Antonia por ser la primera mujer en obtener un doctorado en la Facultad de Filosofía y Letras, en la Universidad de Buenos Aires, con un tema de Antropología Física, así como por su relevancia y su trascendencia internacional. Paola Silvia Raimundo explica, de esta forma, la 
producción de Antonia Juliane Dillenius, la polémica de su temática y la importancia de las mujeres que abrieron la senda a otras para acceder a la universidad como estudiantes y también como profesoras.

Nailda Marinho Costa y Libaina Xavier en «El asociativismo de mujeres en Brasil y las tesis de Branca Canto de Melo en el primer congreso internacional feminista (1922)» discuten el activismo y las concepciones educativas de esta autora de cara a reflexionar sobre la historia de la formación del profesorado en Brasil. A lo largo del texto se desarrolla un debate en torno a los conceptos de género e historia de las mujeres, la creación de la Federación Brasileira por el Progreso Femenino, la realización del Primer Congreso Internacional Femenino, la vida y obra de Branca Canto de Melo y la lucha organizada de las mujeres brasileñas a comienzos del siglo XX.

En el capítulo titulado «Educadoras chilenas que trabajaron por la igualdad educativa para niñas y jovencitas en el siglo XX», Estela Socías Muñoz recoge los aportes educativos de cinco educadoras que mantuvieron una constante e importante preocupación por la educación en su país, y que ayudaron a que hoy en día las niñas tengan pleno acceso al sistema educativo. Amanda Labarca fue la primera mujer latinoamericana en ejercer una cátedra universitaria. Creadora de la Liga Cívica Femenina, abogaba por la educación democrática y laica al tiempo que defendía la independencia económica y espiritual de la mujer. Irma Salas fue Directora General de Educación Secundaria y varias de sus propuestas pedagógicas aún subsisten en el sistema educativo chileno. Teresa Clera Mirtin, mujer católica y directora de la Escuela Normal Rural Femenina, destacó por sus aportaciones a la lecto-escritura en educación preescolar. Viola Soto Guzmán habló sobre la necesidad de adaptar el currículum y la metodología a la idiosincrasia de cada centro y Mabel Condemarín Gimberg luchó por una mejora de la educación especial.

El duodécimo texto, «Entre la tradición y la modernidad. Modelos y discursos educativos de la mujer española en los textos escolares de la Segunda República (1931-1936)», Teresa Rabazas Romero y Carlos Sanz Simón analizan los manuales escolares de este período a fin de conocer qué modelos de mujer se sugerían y cuál era la presencia femenina en ellos. A través de manuales escolares para niñas y textos escolares disciplinares, han podido advertir la coexistencia de diferentes modelos de 
mujer. Entre textos de carácter progresista y conservador, en este momento histórico se difundieron diversos ideales de mujer (santa, intelectual, vinculada al ámbito doméstico, feminista, etc.). Sin embargo, desde la perspectiva de la importancia de los mensajes y las representaciones se recalca cómo, por primera vez, las mujeres comenzaron a escribir y a ser y poner sobre la mesa referentes y figuras fuera de los roles de género tradicionales.

A continuación, da comienzo la tercera parte del libro, la cual trata sobre la segunda mitad del siglo XX y los primeros años del siglo XXI. La inaugura el capítulo que lleva por título «La acción de una mujer destacada en pro de la educación: la pedagoga Beatrice Ávalos y su aporte a la educación en Chile, Gales y Oceanía» de Jaime Caiceo Escudero. Siguiendo la línea de textos anteriores, en este se recupera la biografía, el pensamiento pedagógico y la visión de una educadora importante. Beatrice Ávalos, a lo largo de su vida y en diferentes países del mundo, luchó por la mejora de la situación económica y educativa de las mujeres en los países en desarrollo y se implicó especialmente en la formación del profesorado.

El capítulo número catorce, «¿Qué fue de ellas? Profesoras de instituto depuradas durante el franquismo», está escrito por Isabel Grana Gil. En él realiza un análisis tanto cuantitativo como cualitativo de las trayectorias de mujeres profesoras que fueron sancionadas de una $\mathrm{u}$ otra forma durante el franquismo. La autora destaca cómo la depuración fue menor entre las mujeres, pero la pérdida fue cualitativamente mayor ya que se truncó la independencia y vida libre y pública que comenzaban a tener muchas de ellas. Así, recoge las trayectorias vitales y laborales de Concepción Albornoz Salas, María de los Desamparados de Alcedo y de la Espada, Carmen Ambroj Ineva, María Amigo Amigo, Olimpia Arozena Torres, Isabel Bullido Gómez, Consuelo Burell i Mata, Francisca Bustos Jiménez, Donaciana Cano Iriarte, Carmen Castro Medinaveitia, Dolores Escribano Baonza, Adela Gil Crespo, Pilar Madariaga Rojo, Sara Maynar Escanilla y María Dolores de Palacio Azara.

En «La educación católica femenina y el modo francés de educar de las hermanas de San José de Chambéry en Curitiba, Sur de Brasil, 1946 a 1970", Heloísa H. Daldin Pereira y Ariclê Vechia explican la educación escolar femenina que introdujo esta congregación religiosa siguiendo el 
molde francés del catolicismo. Las autoras analizan cómo se preparaba a las alumnas para la vida en sociedad y el comportamiento adecuado a su estatus como burguesas y al mismo tiempo madres, responsables del mantenimiento del matrimonio, la armonía de la familia y los valores católicos tradicionales. Analizan también el modelo de mujer que transmitían los periódicos para muchachas y que coinciden con el ideal mencionado (virginidad, inocencia, recato, maternidad, amor, comprensión, etc.).

El siguiente capítulo trata de analizar la representación y caracterización que se hace de las mujeres que aparecen en las novelas rosas ambientadas en las Islas Canarias. De esta forma, María Isabel González Cruz y María Jesús Vera-Cazorla muestran la realidad sociocultural y educativa que subyace a estas novelas en un texto titulado "Género e identidad cultural: perfiles femeninos en un corpus de novela rosa». En él presentan la novela como un agente cultural para la transmisión de ideologías, realizan un repaso del lenguaje como constructor y perpetuador de estereotipos de género y ahondan en los modelos de feminidad y masculinidad imperantes en diferentes entornos y culturas.

«Mujeres en transición: una aproximación al entorno asociativo de las mujeres en la España de los setenta» pone en relieve la participación de las mujeres en el proceso transicional "desde abajo», tal y como explica Pamela Radcliff. Desde esta perspectiva, pone el foco en tres clases de asociaciones: el movimiento feminista (que realizaba reivindicaciones de tipo político-jurídico), las Asociaciones de Amas de Casa (que fueron fieles al régimen, pero durante la transición construyeron un espacio propio de debate en el que se apostó por la evolución o reevaluación de los roles femeninos) y las Asociaciones de Vecinos (espacios mixtos que luchaban por la mejora de los barrios, pero crearon secciones femeninas en las que se trataban temas tales como el sexo, el control de la natalidad o las violaciones).

El texto escrito por Lucilene Morandi e Hildete Pereira de Melo, "Mujeres y educación en Brasil: una mirada de género», discute cómo entre 1970 y el siglo XXI, las mujeres superaron la brecha de género en la educación en Brasil a partir de la universalización de la educación. Realiza un análisis histórico sobre la educación de las mujeres en Brasil desde la creación del Estado Nacional y aporta datos segregados por 
sexo que muestran el avance de la sociedad brasileña. Sin embargo, destaca el hecho de que, a pesar de que hoy en día exista un acceso igualitario a la educación secundaria y superior, aún exista una evidente injusticia social que se manifiesta a través del laberinto de cristal o la feminización del amor.

El libro lo cierra el capítulo titulado «La influencia de la educación en la mujer costarricense» de María Lizano DiMare. Este texto se centra en las necesidades, las oportunidades y las barreras de las mujeres en la educación actual de Costa Rica a través de la opinión de 265 estudiantes de centros públicos de entre 15 y 41 años. Así, se ha obtenido información sobre la relevancia de la educación, las cuestiones de género, el currículo, la vida estudiantil y la comunidad. Los resultados de esta investigación aportan información tan interesante como es la necesidad manifiesta de promover y recuperar las aportaciones de las mujeres a las áreas científicas y a la sociedad, o la importancia de crear un currículum que trate la comunicación, el respeto, la tolerancia y la igualdad de género.

Con todo lo expuesto anteriormente, podemos comprobar cómo el libro aporta elementos imprescindibles para la recuperación y visibilización de la labor de las mujeres a lo largo de la historia y, de manera más concreta, en el ámbito de la educación. De esta manera, construye una nueva historia que incluye no solo a las mujeres, sino también los temas, teorías y experiencias que habían sido entendidas como irrelevantes o periféricas para ampliar la visión tradicional que ponía al hombre en el centro del conocimiento.

Ainhoa Resa Ocio

Universidad Complutense de Madrid aresa@ucm.es 